\title{
The importance of masked hypertension in adults with prehypertension
}

Brent M. Egan and Sean Stevens-Fabry

In their Correspondence (Prehypertension or masked hypertension-which is responsible for target-organ damage? Nat. Rev. Cardiol. doi:10.1038/nrcardio.2015.99) ${ }^{1}$ on our Review (Prehypertension-prevalence, health risks, and management strategies. Nat. Rev. Cardiol. 12, 289-300; 2015), ${ }^{2}$ Drs Chen, Huang, and Mai cite evidence indicating that masked hypertension is an important factor in the target-organ damage and clinical risk associated with prehypertension. We concur with the importance of assessing out-of-office blood pressure in patients with prehypertension, and addressed the issue of masked hypertension in our Review, ${ }^{2}$ where we stated that "assessment of blood-pressure values outside the office setting is useful for identifying adults with prehypertension who are at particularly increased risk of progression to hypertension. Those with hypertension outside the office ( $\geq 135 / \geq 85 \mathrm{mmHg}$ ), despite nonhypertensive office blood-pressure values, are at increased risk of incident hypertension and cardiovascular events." We cited evidence that $80 \%$ of individuals with office blood pressure 130$139 / 85-89 \mathrm{mmHg}$ and home systolic blood pressure $\geq 134 \mathrm{mmHg}$ developed hypertension in the office during 4 years of follow-up. ${ }^{3} \mathrm{We}$ stated that "for the subset of individuals with masked hypertension (out-of-office daytime blood pressure $\geq 135 / \geq 85 \mathrm{mmHg}$ ), both the risk of cardiovascular events and the case for pharmacological intervention are increased. Importantly, data from clinical trials to reduce cardiovascular risk in patients with masked hypertension are lacking."2

Masked hypertension is an important, yet challenging, topic for practising clinicians. The phenotype is only moderately stable over time, with some individuals regressing to normal blood pressure outside the office, some progressing to hypertension both in and outside the office, and a subset of individuals continuing to manifest masked hypertension. ${ }^{4,5}$ The prevalence of masked hypertension is similar when defined with ambulatory and home bloodpressure measurements in some, but not all, studies. ${ }^{6,7}$ The prevalence of both masked hypertension and office hypertension seems to be lower with automated office blood pressure than with usual office blood-pressure measurements, ${ }^{8}$ as noted in our Review. ${ }^{2}$

Masked hypertension is associated with increased risk of cardiovascular disease, but that risk is incompletely defined. ${ }^{6}$ Masked hypertension is associated with other risk factors-for example, high normal office blood pressure or stage 2 prehypertension (blood pressure 130-139/85-89 mmHg), male sex, obesity, and diabetes mellitus-that are also associated with cardiovascular risk and progression to hypertension. Investigators in one study reported that masked hypertension is associated with a significant cardiovascular risk only when defined by elevated nighttime blood pressure, and not when defined by elevated daytime blood pressure using $24 \mathrm{~h}$ ambulatory monitoring. ${ }^{9}$

Given what is known about masked hypertension, and what is yet to be defined, the limited clinical guidance in our Review is appropriate. We concur with the importance of this topic, as described in the Correspondence by Drs Chen, Huang, and Mai, ${ }^{1}$ and the importance of further research to guide clinicians with an evidence-based management plan.
Care Coordination Institute, 300 East McBee Avenue, Suite 401, Greenville, SC 29601, USA

(B.M.E., S.S.-F.)

Correspondence to: B.M.E.

began@ccihealth.org

Competing interests

The authors declare no competing interests.

1. Chen, Y., Huang, Y. \& Mai, W. Prehypertension or masked hypertension — which is responsible for target-organ damage? Nat. Rev. Cardiol. http://dx.doi.org/10.1038/ nrcardio.2015.99.

2. Egan, B. M. \& Stevens-Fabry, S. Prehypertension - prevalence, health risks, and management strategies. Nat. Rev. Cardiol. 12, 289-300 (2015).

3. Julius, S. et al. Feasibility of treating prehypertension with an angiotensin receptor blocker. N. Engl. J. Med. 354, 1685-1697 (2006).

4. Hänninen, M. R. Is it practical to search for masked hypertension? J. Hypertens. 3, 144 (2014).

5. Trudel, X., Milot, A. \& Brisson, C. Persistence and progression of masked hypertension: a 5-year prospective study. Int. J. Hypertens. 2013, 836387 (2013).

6. Viera, A. J. et al. Reproducibility of masked hypertension among adults 30 years or older. Blood Press. Monit. 19, 208-215 (2014).

7. Kawabe, H. \& Saito, I. Reproducibility of masked hypertension determined from morning and evening home blood pressure measurements over a 6-month period. Hypertens. Res. 39, 845-851 (2007).

8. Myers, M. G. et al. The conventional versus automated measurement of blood pressure in the office (CAMBO) trial: masked hypertension sub-study. J. Hypertens. 30, 1937-1941 (2012).

9. Hermida, R. C. et al. Sleep-time blood pressure and the prognostic value of isolated office and masked hypertension. Am. J. Hypertens. 25, 397-305 (2012). 